\title{
Covertex Inscribed Triangles of Parabola in Isotropic Plane
}

\section{Covertex Inscribed Triangles of Parabola in Isotropic Plane \\ ABSTRACT}

In the paper the concept of a covertex inscribed triangle of a parabola in an isotropic plane is introduced. It is a triangle inscribed to the parabola that has the centroid on the axis of parabola, i.e. whose circumcircle passes through the vertex of the parabola. We determine the coordinates of the triangle centers, and the equations of the lines, circles and conics related to the triangle.

Key words: isotropic plane, triangle, parabola

MSC2010: 51N25

\section{Motivation}

The following theorem, which can be found in [10], is a well-known fact from the geometry of Euclidean plane: Let $A, B, C$ be three points on a parabola $\mathcal{P}$ different from its vertex and different mutually. These are the equivalent statements:

$1^{0}$ The normal lines to $\mathcal{P}$ at $A, B, C$ are concurrent.

$2^{0}$ The centroid of the triangle $A B C$ lies on the axis of parabola $\mathcal{P}$.

$3^{0}$ The circumcircle of the triangle ABC passes through the vertex of parabola $P$.

The perpendicularity is not defined in the isotropic plane, and often an isotropic line plays a role of a line perpendicular to a given one. Therefore, every normal to $P$ passes through the absolute point. From that point of view, the property $1^{0}$ is fulfilled for any three points on the parabola, and it is interesting to study the triangles having properties $2^{0}$ and $3^{0}$.
Covertex trokuti upisani paraboli u izotropnoj ravnini

\section{SAŽETAK}

$U$ radu se uvodi pojam covertex trokuta upisanog paraboli u izotropnoj ravnini. To je trokut upisan paraboli čije težište leži na osi parabole, tj. čija opisana kružnica prolazi tjemenom parabole. Određuju se koordinate točaka te jednadžbe pravaca, kružnica i konika povezanih s tim trokutom.

Ključne riječi: izotropna ravnina, trokut, parabola

The result above together with other results stated in [10] inspired the authors to write this paper.

\section{Introduction}

Let us start by recalling some basic facts about the isotropic plane. The isotropic plane is a real projective metric plane whose absolute figure is a pair consisting of an absolute point $\Omega$ and an absolute line $\omega$ incident to it, [11], [12]. The isotropic points are the points incident with the absolute line $\omega$ and the isotropic lines are the lines passing through the absolute point $\Omega$.

In the affine model of the isotropic plane where the coordinates of the points are defined by $x=\frac{x_{0}}{x_{2}}, y=\frac{x_{1}}{x_{2}}$, the absolute line has the equation $x_{2}=0$ and the absolute point has the coordinates $(0: 1: 0)$.

Two lines are parallel if they pass through the same isotropic point, and two points are parallel if they are incident with the same isotropic line. For two non-parallel points $A=\left(x_{A}, y_{A}\right)$ and $B=\left(x_{B}, y_{B}\right)$, a distance is defined 
by $d(A, B)=x_{B}-x_{A}$, and for two parallel points, $x_{A}=x_{B}$, a span is defined by $s(A, B)=y_{B}-y_{A}$. For two non-parallel lines $p$ and $q$, given by the equations $y=k_{p} x+l_{p}$ and $y=k_{q} x+l_{q}$, an angle is defined by $\angle(p, q)=k_{q}-k_{p}$. All these quantities are oriented.

A triangle in the isotropic plane is called allowable if non of its sides is an isotropic line. In our study only such triangles are considered.

The midpoint of the points $A$ and $B$ is defined as $M=$ $\left(\frac{x_{A}+x_{B}}{2}, \frac{y_{A}+y_{B}}{2}\right)$, while the bisector of the lines $p$ and $q$ is given by the equation $y=\frac{k_{p}+k_{q}}{2} x+\frac{l_{p}+l_{q}}{2}$.

A normal line to a line $l$ at a point $P$ is the isotropic line $n$ passing through $P$. A distance from $P$ to $l$ is defined as the span $s(N, P)$, where $N$ is the intersection point of $l$ and $n$, i.e. the point on $l$ parallel to $P$.

The classification of conics in the isotropic plane can be found in [2] and [11]. A circle is a conic touching the absolute line $\omega$ at the absolute point $\Omega$. The equation of such a circle is given by

$y=u x^{2}+v x+w, \quad u \neq 0, \quad u, v, w \in \mathbb{R}$.

A parabola is a conic touching the absolute line at a point different from the absolute point. By choosing a suitable coordinate system every parabola can be represented by the equation

$y^{2}=x$.

It has vertex (focus) at $O=(0,0)$, the $x$-axis as its axis, and $y$-axis as its directrix. A numerous properties of parabola were discussed in [13], [15] and [19].

\section{Covertex Inscribed Triangles of Parabola}

Let $A=\left(a^{2}, a\right), B=\left(b^{2}, b\right), C=\left(c^{2}, c\right)$ be three points on the parabola $\mathcal{P}$ given by (1) different from its vertex and different mutually. Thus, $a, b, c$ are mutually different nonzero real numbers. It can be easily checked that $A, B, C$ lie on the circle $\mathcal{C}$ with equation

$$
\begin{aligned}
& (a+b)(b+c)(c+a) y= \\
& -x^{2}+\left(a^{2}+b^{2}+c^{2}+a b+b c+c a\right) x+a b c(a+b+c) .
\end{aligned}
$$

The circle $C$ passes through the vertex $O=(0,0)$ precisely when

$a+b+c=0$.

In that case the centroid $G=\left(\frac{a^{2}+b^{2}+c^{2}}{3}, \frac{a+b+c}{3}\right)$ of the triangle $A B C$ lies on the axis of the parabola $\mathcal{P}$, and we proved:

Theorem 1 A triangle inscribed to a parabola has the centroid on the axis of parabola if and only if its circumcircle passes through the vertex of parabola.

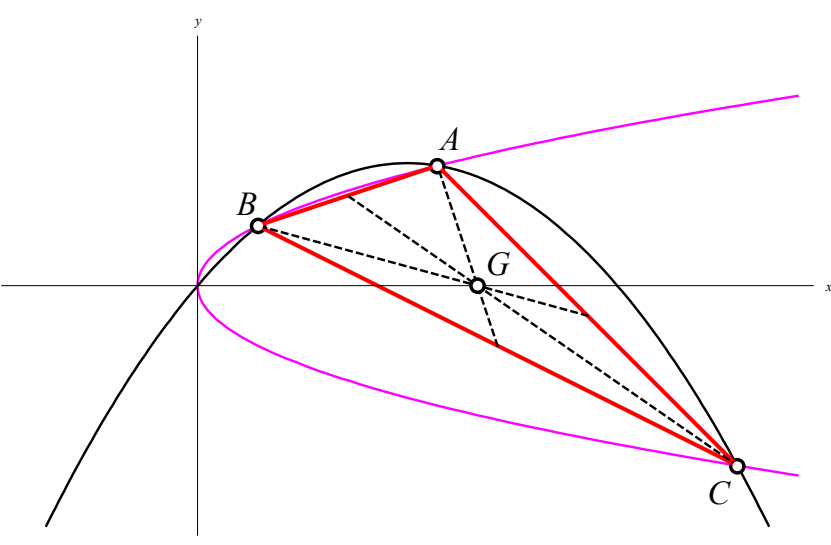

Figure 1: Covertex inscribed triangle of a parabola

The triangles having the property from Theorem 1 are called the covertex inscribed triangles of the parabola. If such a triangle $A B C$ has vertices $A=\left(a^{2}, a\right), B=\left(b^{2}, b\right)$, $C=\left(c^{2}, c\right)$, the equations of its sides are:

$$
\begin{array}{lll}
A B & \ldots & x+c y+a b=0 \\
B C & \ldots & x+a y+b c=0 \\
C A & \ldots & x+b y+c a=0
\end{array}
$$

Considering (3) we will use the standard notation:

$p=a b c, \quad q=a b+b c+c a$.

The following relations are interesting, useful and their validity can easily be proved:

$a^{2}=b c-q$,

$a^{3}=p-a q$,

$a^{2}+b^{2}+c^{2}=-2 q$,

$a^{2} b^{2}+b^{2} c^{2}+c^{2} a^{2}=q^{2}$,

$a^{4}+b^{4}+c^{4}=2 q^{2}$.

The equation (2) of the circumcircle $C$ of $A B C$ now takes the form

$p y=x^{2}+q x$,

the centroid is $G=\left(-\frac{2}{3} q, 0\right)$, and the Euler line has the equation $x=-\frac{2}{3} q$, [6].

The properties of an allowable triangle in the isotropic plane have been studied in numerous papers, [3], [4], [5], [6], [7], [8], [14], [16], [18]. The observed triangle was settled in the standard position having the circumcircle with the equation $y=x^{2}$ and the vertices of the form $A=\left(a, a^{2}\right)$, $B=\left(b, b^{2}\right), C=\left(c, c^{2}\right)$, where $a+b+c=0$. The same notations $p=a b c, q=a b+b c+c a$ were used as well. Therefore, it is not of our interest to prove the theorems on the 
special case of covertex inscribed triangles of parabola. Instead we determine the coordinates of the triangle centers, and the equations of the lines, circles and conics related to the triangles.

The midpoint $A_{m}$ of the points $B$ and $C$ has the coordinates $A_{m}=\left(-q-\frac{1}{2} a^{2},-\frac{1}{2} a\right)$ and lies on the circle with the equation

$p y=-2 x^{2}-3 q x-q^{2}$.

Indeed,

$$
\begin{aligned}
& -2\left(q+\frac{1}{2} a^{2}\right)^{2}+3 q\left(q+\frac{1}{2} a^{2}\right)-q^{2} \\
& =-\frac{1}{2} a^{4}-\frac{1}{2} a^{2} q=-\frac{1}{2} a\left(a^{3}+q a\right)=-\frac{1}{2} a p .
\end{aligned}
$$

It can be proved similarly that the midpoints $B_{m}, C_{m}$ of the sides $A C, A B$, respectively, also lie on the circle given by (7). Thus, that circle is the Euler circle of the triangle $A B C$. Let us now prove that the circle having the equation

$4 p y=x^{2}$

is the inscribed circle of the triangle $A B C$. The line $B C$ has the equation $x+a y+b c=0$ given in (4). The intersection point of $B C$ and the circle (8) is the point $D=\left(-2 b c, \frac{b c}{a}\right)$ having the intersection multiplicity 2 . Therefore, $D$ is the contact point of $B C$ and (8). In the similar way, it can be shown that the lines $C A$ and $A B$ also touch the circle given by (8).

The inscribed circle and Euler circle of a triangle touch each other externally in a point called Feuerbach point, and their common tangent is called the Feuerbach line of the considered triangle, [1]. So, we prove:

Theorem 2 The Feuerbach point of the covertex inscribed triangle $A B C$ of the parabola $P$ is

$\Phi=\left(-\frac{2}{3} q, \frac{1}{9 p} q^{2}\right)$

and its Feuerbach line is given by the equation

$3 q x+9 p y+q^{2}=0$.

Proof. By eliminating $y$ in (7) and (8), the equation $9 x^{2}+12 q x+4 q^{2}=0$ with double root $x=-\frac{2}{3} q$ is obtained. From (8) we get $y=\frac{1}{9 p} q^{2}$. The radical axis of the inscribed circle and Euler circle is obtained by eliminating the terms next to $x^{2}$ in (7) and (8). Thus, the line having the equation $3 q x+9 p y+q^{2}=0$ is their common tangent.

In the isotropic plane the principle of projective duality is preserved. The dual of the triangle inscribed circle is its circumcircle, while the dual of the Euler circle is the circle inscribed to the triangle formed by angle bisectors of the considered triangle, [7]. The circumscribed circle and the dual Euler circle of the triangle touch each other at one point, so-called dual Feuerbach point, and their common tangent is called dual Feuerbach line.

Theorem 3 The dual Feuerbach point of the covertex inscribed triangle $A B C$ of the parabola $P$ is

$\Phi^{\prime}=\left(-\frac{2}{3} q,-\frac{2}{9 p} q^{2}\right)$

and its dual Feuerbach line is given by the equation

$3 q x+9 p y+4 q^{2}=0$.

Proof. The equations of the sides $C A, A B$ from (4) can be written in the form $c x+b c y+a c^{2}=0, b x+b c y+a b^{2}=0$, and their angle bisector has equation $-a x+2 b c y+a\left(b^{2}+\right.$ $\left.c^{2}\right)=0$, which because of $-b^{2}-c^{2}=q+b c$, and after multiplying by $a$, turns to $2 p y=a^{2} x+a^{2} q+a p$. By eliminating the terms next to $x^{2}$ in this equation and in the equation of the circle

$8 p y=-x^{2}-4 q x-4 q^{2}$

we obtain the equation $x^{2}+4\left(a^{2}+q\right) x+4\left(a^{2} q+q^{2}+\right.$ $a p)=0$. Because of

$$
a^{2} q+q^{2}+a p=b c q+a^{2} b c=b^{2} c^{2}
$$

it turns to the equation $x^{2}+4 b c x+4 b^{2} c^{2}=0$ having a double root. Therefore, the observed angle bisector touches the circle (13). The same fact holds for the other two angle bisectors of the triangle $A B C$ as well. Thus, (13) is the equation of the dual Euler circle of $A B C$. Now by eliminating $y$ in (6) and (13) we get equation $9 x^{2}+12 q x+$ $4 q^{2}=0$ with a double root $x=-\frac{2}{3} q$. Hence, the point $\left(-\frac{2}{3} q,-\frac{1}{9 p} q^{2}\right)$ is their contact point. By eliminating the terms next to $x^{2}$ in (6) and (13), the equation (12) of their radical axis is obtained.

The orthic axis of the triangle is the radical axis of a pencil of circles consisting of some important circles of that triangle as the circumcircle, Euler circle and polar circle, see [1] and [14].

Theorem 4 The orthic axis of the covertex inscribed triangle $A B C$ of the parabola $P$ is given by the equation

$q x+3 p y+q^{2}=0$.

Proof. By eliminating the terms next to $x^{2}$ in the equations (6) and (7) of the circumcircle and Euler circle of the triangle $A B C$, the equation (14) of their radical axis is obtained. 
Theorem 5 The polar circle of the covertex inscribed triangle $A B C$ of the parabola $P$ has the equation

$2 p y=-x^{2}-2 q x-q^{2}$

Proof. The polar of the point $(u, v)$ with respect to the circle (15) has the equation $p(y+v)=-x u-q(x+u)-q^{2}$. Therefore, the polar of $A=\left(a^{2}, a\right)$ has the equation $p y+$ $\left(q+a^{2}\right) x+\left(q+a^{2}\right) q+a p=0$, i.e. $p y+b c x+b c\left(q+a^{2}\right)=$ 0 , and finally $x+a y+b c=0$ being the equation of the side $B C$. Similarly we get that the polars of $B$ and $C$ are the sides $C A$ and $A B$, respectively.

The circles of the pencil mentioned above intersect each other in the points having coordinates

$$
(-q, 0), \quad\left(-\frac{q}{3},-\frac{2}{3 p} q^{2}\right)
$$

This can be easily proved by using e.g. (6) and (7).

The Gergonne point of the triangle $A B C$ is the intersection point of three cevians $A D, B E, C F$ defined by the contact points $D, E, F$ of the inscribed circle, [3], and we have:

Theorem 6 The Gergonne point of the covertex inscribed triangle $A B C$ of the parabola $\mathcal{P}$ has the coordinates

$\Gamma=\left(\frac{9 p^{2}}{q^{2}},-\frac{3 p}{q}\right)$

Proof. The line with the equation $q x+(3 p-a q) y=3 a p$ obviously passes through the vertex $A=\left(a^{2}, a\right)$. It also passes through the contact point $D=\left(-2 b c, \frac{b c}{a}\right)$ of the side $B C$ and the inscribed circle of the triangle $A B C$ since

$$
-2 b c q+3 b^{2} c^{2}-b c q=3 b c(b c-q)=3 a p .
$$

Thus, the observed line is the cevian $A D$. On the other hand, it also passes through the point $\Gamma$ given in (16). Since the analogue statements hold for the lines $B E$ and $C F, \Gamma$ is the Gergonne point of the triangle $A B C$.

Corollary 1 The Gergonne point of the covertex inscribed triangle $A B C$ of the parabola $\mathcal{P}$ lies on the parabola $\mathcal{P}$

Corollary 1 together with Theorem 1 present new geometric results in the isotropic plane.

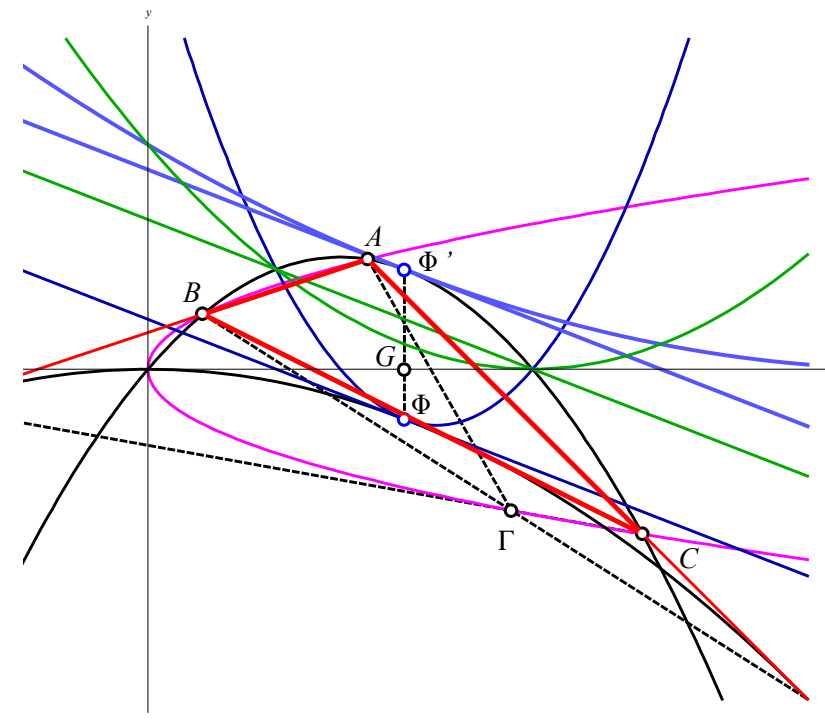

Figure 2: The centroid $G$, Feuerbach point $\Phi$, dual Feuerbach point $\Phi^{\prime}$ and Gergonne point $\Gamma$ of the covertex inscribed triangle $A B C$ of a parabola

Theorem 7 Every conic circumscribed to the covertex inscribed triangle $A B C$ of the parabola $\mathcal{P}$ has the equation of the form

$A x^{2}+2 B x y+C y^{2}+(q A-C) x+(2 q B-p A) y-2 p B=0$,

the axis with equation

$B x+C y+q B-\frac{1}{2} p A=0$

and the center with the coordinates

$M=\left(\frac{2 q B^{2}-p A B-q A C+C^{2}}{2\left(A C-B^{2}\right)}, \frac{p A^{2}-q A B-B C}{2\left(A C-B^{2}\right)}\right)$.

Proof. Every conic is given by the equation of the form

$A x^{2}+2 B x y+C y^{2}+2 D x+2 E y+F=0$.

The points $A=\left(a^{2}, a\right)$ and $B=\left(b^{2}, b\right)$ lie on the conic precisely when the following equalities hold:

$$
\begin{aligned}
& a^{4} A+2 a^{3} B+a^{2} C+2 a^{2} D+2 a E+F=0, \\
& b^{4} A+2 b^{3} B+b^{2} C+2 b^{2} D+2 b E+F=0 .
\end{aligned}
$$

By subtracting the two upper equations and dividing by $a-b$ we get

$$
\begin{aligned}
& \left(a^{2}+b^{2}\right)(a+b) A+2\left(a^{2}+a b+b^{2}\right) B+(a+b) C \\
& \quad+2(a+b) D+2 E=0
\end{aligned}
$$


i.e.

$(c q+p) A-2 q B-c C-2 c D+2 E=0$

since

$$
\left(a^{2}+b^{2}\right)(a+b)=(-q-a b)(-c)=c q+p .
$$

Similarly, we get

$$
\begin{aligned}
& (b q+p) A-2 q B-b C-2 b D+2 E=0 \\
& (a q+p) A-2 q B-a C-2 a D+2 E=0 .
\end{aligned}
$$

It immediately follows $p A-2 q B+2 E=0$, and from (22) also $q A-C-2 D=0$. Thus, $2 D=q A-C$ and $2 E=2 q B-p A$ are valid. From (20) we get $\left(a^{4}+a^{2} q-\right.$ $a p) A+2\left(a^{3}+a q\right) B+F=0$, and finally $F=-2 p B$ since $a^{3}+a q=p$. We proved that the conic circumscribed to the triangle $A B C$ has to have the equation of the form (17).

The equation (20) can be written in homogeneous coordinates as follows

$$
A x_{0}^{2}+2 B x_{0} x_{1}+C x_{1}^{2}+2 D x_{0} x_{2}+2 E x_{1} x_{2}+F x_{2}^{2}=0,
$$

and the point $\left(u_{0}: u_{1}: u_{2}\right)$ has the polar with the equation

$$
\begin{aligned}
& A u_{0} x_{0}+B\left(u_{1} x_{0}+u_{0} x_{1}\right)+C u_{1} x_{1}+D\left(u_{0} x_{2}+u_{2} x_{0}\right) \\
& \quad+E\left(u_{1} x_{2}+u_{2} x_{1}\right)+F u_{2} x_{2}=0 .
\end{aligned}
$$

Particularly, the polar of the absolute point $\Omega=(0: 1: 0)$ is given by the equation $B x_{0}+C x_{1}+E x_{2}=0$, i.e. in affine coordinates $B x+C y+E=0$. Thus, when the conic is given by the equation (17), its axis is given by the equation (18). The equation (23) written in the form

$$
\begin{gathered}
\left(A u_{0}+B u_{1}+D u_{2}\right) x_{0}+\left(B u_{0}+C u_{1}+E u_{2}\right) x_{1} \\
+\left(D u_{0}+E u_{1}+F u_{2}\right) x_{2}=0
\end{gathered}
$$

is the equation of the absolute line $x_{2}=0$ precisely when $A u_{0}+B u_{1}+D u_{2}=0$ and $B u_{0}+C u_{1}+E u_{2}=0$. Therefore, the coordinates of the point $M=\left(\frac{u_{0}}{u_{2}}, \frac{u_{1}}{u_{2}}\right)=(u, v)$ have to satisfy the equations $A u+B v+D=0$ and $B u+C v+E=0$, which leads to

$$
M=\left(\frac{B E-C D}{A C-B^{2}}, \frac{B D-A E}{A C-B^{2}}\right) .
$$

So, when the conic is given by (17), its center is given by (19).

The properties of the Steiner ellipse, Steiner axis and Steiner point of a triangle in the isotropic plane were studied in details in [8] and [18]. Here we prove the following two theorems:
Theorem 8 The Steiner ellipse of the covertex inscribed triangle $A B C$ of the parabola $P$ has the equation

$3 q x^{2}+9 p x y-q^{2} y^{2}+4 q^{2} x+6 p q y-9 p^{2}=0$,

while the Steiner axis is given by

$9 p x-2 q^{2} y+6 p q=0$.

Proof. By inserting $A=3 q, B=\frac{9}{2} p, C=-q^{2}$ into (17), (18), (19), the circumscribed conic (24) with the axis (25) and the center $M=\left(-\frac{2}{3}, 0\right)$ is obtained. The point $M$ is the centroid $G$ of the triangle $A B C$, and therefore (24) is the equation of the Steiner ellipse.

Theorem 9 The Steiner point of the covertex inscribed triangle $A B C$ of the parabola $P$ has the coordinates

$S=\left(\frac{9 p^{2}}{q^{2}}, \frac{81 p^{3}}{q^{4}}+\frac{9 p}{q}\right)$.

Proof. The coordinates of the point $S$ satisfy the equations (6) and (24). So, it is the intersection of the circumcircle and the Steiner ellipse of the triangle $A B C$.

The isogonal conjugate $P^{\prime}$ of a point $P$ with respect to triangle $A B C$ is constructed by reflecting the lines $A P, B P$, $C P$ about the angle bisectors at the points $A, B, C$, respectively. The three reflected lines then concur at the isogonal conjugate $P^{\prime}$. The isogonality in the isotropic plane was studied in [9].

Theorem 10 The isogonal point $P^{\prime}=\left(x^{\prime}, y^{\prime}\right)$ of the point $P=(x, y)$ with respect to the covertex inscribed triangle $A B C$ of the parabola $\mathcal{P}$ has the coordinates

$$
\begin{aligned}
x^{\prime} & =-\frac{q x^{2}+p x y+q^{2} x-p^{2}}{x^{2}+q x-p y}, \\
y^{\prime} & =\frac{q x y+p y^{2}-p x+q^{2} y-p q}{x^{2}+q x-p y} .
\end{aligned}
$$

Proof. If a point $P=(u, v)$ is given, then the slope of the line $A P$ equals to $k=\frac{v-a}{u-a^{2}}$. The line isogonal to the line $A P$ with respect to the lines $A B$ and $A C$ with slopes $-\frac{1}{c}$ and $-\frac{1}{b}$, respectively, has the slope

$$
-\frac{1}{c}-\frac{1}{b}-k=\frac{a}{b c}-\frac{v-a}{u-a^{2}}=\frac{a u-b c v+a q}{b c u-a p} .
$$

and the equation

$$
(b c v-a u-a q) x+(b c u-a p) y=a q u+a p v-a^{2} p-a^{3} q .
$$

Similarly, the line isogonal to the line $B P$ with respect to the lines $B C$ and $B A$ has the equation

$$
(c a v-b u-b q) x+(c a u-b p) y=b q u+b p v-b^{2} p-b^{3} q .
$$


By subtracting (28) and (29) and then dividing by $a-b$ we get

$$
(c v+u+q) x+(c u+p) y=-q u-p v-c p-q^{2} .
$$

So, we conclude that the following two equalities have to hold

$$
(u+q) x+p y=-q u-p v-q^{2}, \quad v x+u y=-p .
$$

Solving the system of equations, we get

$x=-\frac{q u^{2}+p u v+q^{2} u-p^{2}}{u^{2}+q u-p v}, y=\frac{q u v+p v^{2}-p u+q^{2} v-p q}{u^{2}+q u-p v}$,

which finishes the proof.

The symmedian center $K$ is the isogonal conjugate of the triangle centroid $G$. Therefore we have:

Theorem 11 The symmedian center $K$ of the covertex inscribed triangle $A B C$ of the parabola $\mathcal{P}$ has the coordinates

$K=\left(-\frac{2 q^{3}+9 p^{2}}{2 q^{2}}, \frac{3 p}{2 q}\right)$

and the Brocard diameter is given by

$x=-\frac{2 q^{3}+9 p^{2}}{2 q^{2}}$.

Proof. The claim follows from (27) after inserting $x=$ $-\frac{2}{3} q, y=0$.

Theorem 12 The isogonal image of the isotropic line $x^{\prime}=$ $m$ with respect to the covertex inscribed triangle $A B C$ of the parabola $\mathcal{P}$ is a special hyperbola circumscribed to $A B C$ having equation

$(q+m) x^{2}+p x y+q(q+m) x-m p y-p^{2}=0$.

Proof. It follows directly from the first equation in (27).

The isogonal image of the Euler line, so-called Jeřabek hyperbola, and the isogonal image of the Brocard diameter, so-called Kiepert hyperbola, in the case of standard triangle were studied in detailed in [5] and [16]. Here we prove

Theorem 13 The Jerrabek hyperbola of the covertex in scribed triangle $A B C$ of the parabola $\mathcal{P}$ is given by

$q x^{2}+3 p x y+q^{2} x+2 p q y-3 p^{2}=0$,

while its Kiepert hyperbola is given by

$9 p x^{2}-2 q^{2} x y+9 p q x-\left(2 q^{3}+9 p^{2}\right) y+2 p q^{2}=0$.

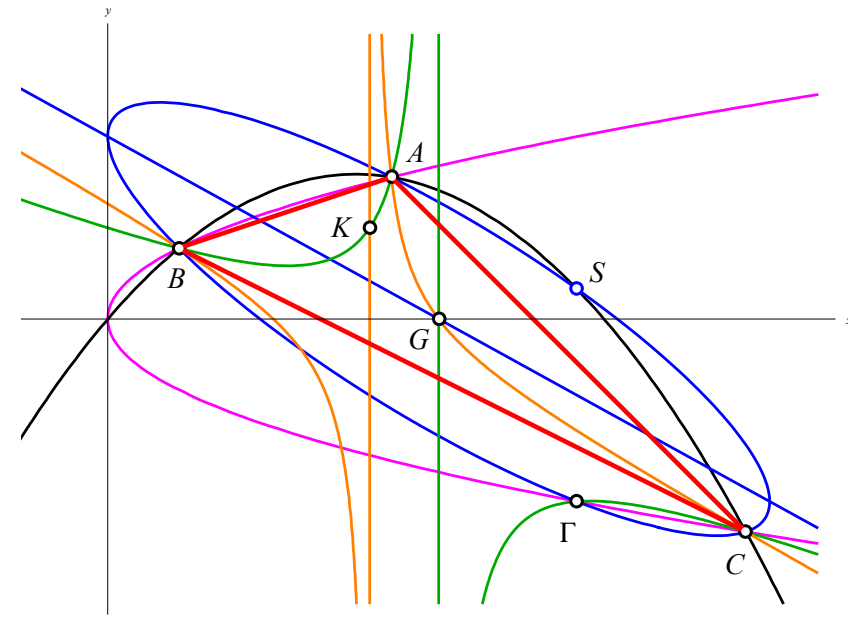

Figure 3: The centroid $G$, symmedian center $K$ and Steiner point $S$ of the covertex inscribed triangle ABC of a parabola. The Steiner axis and Steiner ellipse are marked in blue, the Euler line and Jeřabek hyperbola are marked in green, while the Brocard diameter and Kiepert hyperbola are marked in orange.

Proof. The equations (32) and (33) follow directly from Theorem 12 by inserting $m=-\frac{2}{3} q$ and $m=-\frac{2 q^{3}+9 p^{2}}{2 q^{2}}$ into (31) for the Euler line and Brocard diameter, respectively.

The concept of reciprocity with respect to the standard triangle in the isotropic plane was introduced and studied in [17]. Here we prove the following theorem:

Theorem 14 The lines given by the equations

$U x+V y+W=0$

and

$U^{\prime} x+V^{\prime} y+W^{\prime}=0$,

where

$U^{\prime}=q U^{2}+V^{2}-U W, \quad V^{\prime}=p U^{2}-V W$,

$W^{\prime}=q^{2} U^{2}+q V^{2}+W^{2}-p U V-2 q U W$,

are reciprocal lines with respect to the covertex inscribed triangle $A B C$ of the parabola $\mathcal{P}$.

Proof. Let the line having the equation (34) be given. The points

$D=\left(\frac{b c V-a W}{a U-V}, \frac{W-b c U}{a U-V}\right)$,
$D^{\prime}=\left(\frac{q V+a W-a q U-p U}{a U-V}, \frac{q U+a V-W}{a U-V}\right)$ 
lie on the line $B C$ given by $x+a y+b c=0$, and they are isotomic conjugate since they have the same midpoint as the points $B$ and $C$. Indeed,

$$
\begin{aligned}
& \frac{b c V-a W}{a U-V}+\frac{q V+a W-a q U-p U}{a U-V}=-q-b c=b^{2}+c^{2}, \\
& \frac{W-b c U}{a U-V}+\frac{q U+a V-W}{a U-V}=-a=b+c .
\end{aligned}
$$

The point $D$ obviously lies on the line (34), and point $D^{\prime}$ on the line (35). Thus, the lines (34) and (35) intersect the line $B C$ in the isotomic conjugate points of the side $B C$. The similar claims can be proved for the sides $A B$ and $C A$ as well. Therefore, the lines (34) and (35) are reciprocal with respect to the triangle $A B C$.

Using Theorem 14 it can be easily shown that the orthic axis of the triangle $A B C$ is reciprocal to its Steiner axis, while the Euler line of the triangle is reciprocal to its Feuerbach line.

The concepts of equicevian points and equiangular lines of the triangle in the isotropic plane were studied in [4] where a number of their significant properties was considered. A point $P$ such that the points $D=A P \cap B C, E=B P \cap C A$, $F=C P \cap A B$ satisfy the equalities

$d(A, D)=d(B, E)=d(C, F)=t^{2}, \quad t \neq 0$,

is called the equicevian point of the triangle $A B C$. It is known that each allowable triangle in the isotropic plane has two equicevian points.

Theorem 15 The equicevian points of the covertex inscribed triangle $A B C$ of the parabola $\mathcal{P}$ are of the form

$P=\left(\frac{2}{3}\left(t^{2}-q\right), \frac{3 p}{t^{2}}\right)$

where $t^{2}= \pm q$.

Proof. Let us look for a point $P$ such that (38) is fulfilled. The abscissa of the point $D$ equals $a^{2}+t^{2}$, and the equality

$$
\frac{d(B, D)}{d(C, D)}=\frac{t^{2}+a^{2}-b^{2}}{t^{2}+a^{2}-c^{2}}
$$

follows. Similarly we get

$$
\frac{d(C, E)}{d(A, E)}=\frac{t^{2}+b^{2}-c^{2}}{t^{2}+b^{2}-a^{2}}, \quad \frac{d(A, F)}{d(B, F)}=\frac{t^{2}+c^{2}-a^{2}}{t^{2}+c^{2}-b^{2}} .
$$

According to Ceva's theorem, which also holds in the isotropic plane, the lines $A D, B E$ and $C F$ pass through the point $P$ if and only if $d(B, D) d(C, E) d(A, F)$ $+d(C, D) d(A, E) d(B, F)=0$, i.e.

$$
\begin{aligned}
& \left(t^{2}+a^{2}-b^{2}\right)\left(t^{2}+b^{2}-c^{2}\right)\left(t^{2}+c^{2}-a^{2}\right) \\
& \quad+\left(t^{2}+a^{2}-c^{2}\right)\left(t^{2}+b^{2}-a^{2}\right)\left(t^{2}+c^{2}-b^{2}\right)=0
\end{aligned}
$$

which holds precisely when

$$
2 t^{6}+2 t^{2}\left(b^{2} c^{2}+c^{2} a^{2}+a^{2} b^{2}-a^{4}-b^{4}-c^{4}\right)=0,
$$

i.e.

$$
2 t^{6}-2 q^{2} t^{2}=0
$$

As $t \neq 0$, it has to be $t^{4}-q^{2}=0$, which leads to $t^{2}= \pm q$. The line $B C$ has the equation $y=-\frac{1}{a} x-\frac{b c}{a}$, so the ordinate of the point $D$ equals $\frac{1}{a}\left(-a^{2}-t^{2}-b c\right)=\frac{1}{a}\left(q-2 b c-t^{2}\right)$. Therefore,

$$
D=\left(a^{2}+t^{2}, \frac{1}{a}\left(q-2 b c-t^{2}\right)\right) .
$$

It can be easily checked that the coordinates of the points $A$ and $D$ satisfy the equation

$$
\left(t^{2}-2 q+3 b c\right) x+a t^{2} y=2 a^{2} t^{2}-2 a^{2} q+3 a p .
$$

Thus, that is the equation of the line $A D$. Some short calculation shows that the same line passes through the point $P=\left(\frac{2}{3}\left(t^{2}-q\right), \frac{3 p}{t^{2}}\right)$. The equivalent statements holds for the lines $B E$ and $C F$. Thus the point $P$ is equicevian point of the triangle $A B C$.

The equicevian points of the standard triangle considered in [4] are so to say equal. It is not like that in the case of the covertex inscribed triangle of the parabola. Here the equicevian points are given by the coordinates

$P_{1}=\left(0, \frac{3 p}{q}\right), \quad P_{2}=\left(-\frac{4 q}{3},-\frac{3 p}{q}\right)$.

They are symmetrical with respect to the centroid $G$ of the triangle and they lie on its Steiner axis. It is also known that they are the foci of its circumscribed Steiner ellipse of the triangle $A B C$, [4]. But in the case of the covertex inscribed triangle, it can be noticed that one of them, the point $P_{1}$, lies on the vertex tangent (directrix) of parabola $\mathcal{P}$. So, an interesting geometric fact can be stated:

Theorem 16 The circumscribed Steiner ellipse of the covertex inscribed triangle ABC of the parabola $\mathcal{P}$ touches the directrix of parabola $P$ at one of the foci.

A line $l$ such that the points $D^{\prime}=l \cap B C, E^{\prime}=l \cap C A$, $F^{\prime}=l \cap A B$ satisfy the equalities

$\angle\left(B C, A D^{\prime}\right)=\angle\left(C A, B E^{\prime}\right)=\angle\left(A B, C F^{\prime}\right)=\varphi, \quad \varphi \neq 0$,

is called the equiangular line of the triangle $A B C$. It was proved in [4] that each allowable triangle has two equiangular lines. 
Theorem 17 The equiangular lines of the covertex inscribed triangle $A B C$ of the parabola $\mathcal{P}$ have the equations

$q x-3 p y+q^{2}+\frac{9}{q} p^{2}=0, \quad q x+p y+q^{2}+\frac{3}{q} p^{2}=0$.

Proof. Because of (41) the line $A D^{\prime}$ has the slope $\varphi-\frac{1}{a}$, and its equation is

$y=\left(\varphi-\frac{1}{a}\right) x+2 a-a^{2} \varphi$.

From (43) and the equation $y=-\frac{1}{a} x-\frac{b c}{a}$ of the line $B C$ we get the coordinates of the point $D^{\prime}$

$x_{D^{\prime}}=\frac{1}{a \varphi}\left(a^{3} \varphi-2 a^{2}-b c\right)$,

$y_{D^{\prime}}=\frac{1}{a^{2} \varphi}\left(2 a^{2}+b c-a^{3} \varphi-p \varphi\right)$.

Analogously, we obtain the coordinates of the point $E^{\prime}$

$x_{E^{\prime}}=\frac{1}{b \varphi}\left(b^{3} \varphi-2 b^{2}-c a\right)$,

$y_{E^{\prime}}=\frac{1}{b^{2} \varphi}\left(2 b^{2}+c a-b^{3} \varphi-p \varphi\right)$.

Using the equalities $a+b=-c$ and $a^{2}+a b+b^{2}=-q$, the slope of the line $D^{\prime} E^{\prime}$ is calculated

$$
\frac{1}{a b} \cdot \frac{a^{2} b^{2} \varphi+c p \varphi-c q}{2 a b+c^{2}+p \varphi}=\frac{1}{p} \cdot \frac{a b p \varphi+c^{2} p \varphi-c^{2} q}{2 a b+c^{2}+p \varphi} .
$$

Similarly, we determine the slope of the line $D^{\prime} F^{\prime}$

$$
\frac{1}{p} \cdot \frac{a c p \varphi+b^{2} p \varphi-b^{2} q}{2 a c+b^{2}+p \varphi} .
$$

The points $D^{\prime}, E^{\prime}$ and $F^{\prime}$ are collinear precisely when

$$
\frac{a b p \varphi+c^{2} p \varphi-c^{2} q}{2 a b+c^{2}+p \varphi}-\frac{a c p \varphi+b^{2} p \varphi-b^{2} q}{2 a c+b^{2}+p \varphi}=0,
$$

which is equivalent to $p^{2} \varphi^{2}-q^{2}=0$. Thus,

$\varphi= \pm \frac{q}{p}$

Therefore, the slope of the line $D^{\prime} E^{\prime}$ equals

$$
\frac{1}{p} \cdot \frac{ \pm a b q \pm c^{2} q-c^{2} q}{2 a b+c^{2} \pm q}=\frac{1}{p} \cdot \frac{ \pm 2 a b q \mp q^{2}-a b q+q^{2}}{3 a b-q \pm q} .
$$

So, we get two lines with slopes $\frac{q}{3 p}$ and $-\frac{q}{p}$. Since equiangular lines of a triangle pass through its symmedian center, [4], which is now given by (30), their equations are of the form (42).

\section{References}

[1] J. BEBAN-BRKIĆ, R. KOLAR-ŠUPER, V. Volenec, Z. Kolar-Begović, On Feuerbach's Theorem and a Pencil of Circles in the Isotropic Plane, J. Geom. Graph. 10 (2006), 125-132.

[2] J. BebAn-BRkić, M. Šimić, V. Volenec, On Foci and Asymptotes of Conics in Isotropic Plane, Sarajevo J. Math. 3 (16) (2007), 257-266.

[3] J. Beban-Brkić, V. Volenec, Z. KolarBEgOVIĆ, R. KOLAR-ŠUPER, On Gergonne point of the triangle in isotropic plane, Rad Hrvat. Akad. Znan. Umjet. Mat. Znan. 515 (2013), 95-106.

[4] Z. Kolar-Begović, R. Kolar-ŠUPer, V. VoLENEC, Equicevian points and equiangular lines of a triangle in an isotropic plane, Sarajevo J. Math. 11 (2015), 101-107.

[5] Z. Kolar-Begović, R. Kolar-ŠUPer, V. VoLENEC, Jeřabek Hyperbola of a Triangle in an Isotropic Plane, KoG 22 (2018), 12-19.

[6] R. Kolar-Šuper, Z. Kolar-Begović, V. VoLENEC, J. BEBAN-BRKIĆ, Metrical relationships in standard triangle in an isotropic plane, Math. Commun. 10 (2005), 149-157.

[7] R. Kolar-ŠUper, Z. Kolar-Begović, V. VoLENEC, Dual Feuerbach theorem in an isotropic plane, Sarajevo J. Math. 6 (2010), 109-115.

[8] R. Kolar-Šuper, Z. Kolar-Begović, V. VoLENEC, Steiner point of a triangle in an isotropic plane, Rad Hrvat. Akad. Znan. Umjet. Mat. Znan. 20 (528) (2016), 83-95.

[9] R. Kolar-ŠUper, Z. Kolar-Begović, V. VoLENEC, J. BEBAN-BRKIĆ, Isogonality and inversion in an isotropic plane, Int. J. Pure Appl. Math. 44 (3) (2008), 339-346.

[10] J. Plane, Recherches géométriques sur les normales a une parabole issues d'un même point, Nouv. Ann. Math. 11 (1911), 241-261.

[11] H. SACHS, Ebene isotrope Geometrie, ViewegVerlag, Braunschweig-Wiesbaden, 1987.

[12] K. Strubecker, Geometrie in einer isotropen Ebene, Math. Naturwiss. Unterr. 15 (1962-63), $297-$ 306, 343-351, 385-394. 
[13] M. ŠImić Horvath, V. Volenec, J. BebanBRKIĆ, On parabolas related to cyclic quadrangle in isotropic plane, Rad Hrvat. Akad. Znan. Umjet. Mat. Znan. 20 (528) (2016), 97-107.

[14] V. VOLEnEC, J. BEBAN-Brkić, R. KOlarŠUPER, Z. KOLAR-BEGOVIĆ, Orthic axis, Lemoine line and Longchamp's line of the triangle in $I_{2}, \mathrm{Rad}$ Hrvat. Akad. Znan. Umjet. Mat. Znan. 503 (2009), 13-19.

[15] V. Volenec, E. JuRkin, M. ŠImić Horvath, Parabola in Isotropic Plane, manuscript

[16] V. Volenec, Z. Kolar-Begović, R. KolarŠUPER, Kiepert hyperbola in an isotropic plane, Rad Hrvat. Akad. Znan. Umjet. Mat. Znan. 22 (534) (2018), 129-143.

[17] V. Volenec, Z. Kolar-Begović, R. KolarŠUPER, Reciprocity in an isotropic plane, Rad Hrvat. Akad. Znan. Umjet. Mat. Znan. 519 (2014), 171-181.

[18] V. Volenec, Z. Kolar-Begović, R. KolarŚUPER, Steiner's ellipses of the triangle in an isotropic plane, Math. Pannon. 21 (2) (2010), 229238.
[19] V. Volenec, M. Šimić Horvath, E. JuRkin, Circles of curvature at points of parabola in isotropic plane, manuscript

\section{Vladimir Volenec}

e-mail: volenec@math.hr

Faculty of Science, University of Zagreb

Bijenička cesta 30, HR-10000 Zagreb, Croatia

\section{Ema Jurkin}

orcid.org/0000-0002-8658-5446

e-mail: ema.jurkin@rgn.hr

Faculty of Mining, Geology and Petroleum Engineering, University of Zagreb

Pierottijeva 6, HR-10000 Zagreb, Croatia

\section{Marija Šimić Horvath}

orcid.org/0000-0001-9190-5371

e-mail: msimic@arhitekt.hr

Faculty of Architecture, University of Zagreb

Kačićeva 26, HR-10000 Zagreb, Croatia 